Bangladesh J. Plant Taxon. 13(2): 155-170, 2006 (December)

\title{
A PRELIMINARY CHECKLIST OF THE ANGIOSPERMIC FLORA OF GHAGOTIA UNION UNDER KAPASIA UPAZILA IN GAZIPUR DISTRICT, BANGLADESH
}

\author{
Mohammad Shah Alam, Md. Abul Hassan and Mohammad Zashim Uddin ${ }^{1}$ \\ Department of Botany, University of Dhaka, Dhaka-1000, Bangladesh
}

Key words: Preliminary checklist, Angiospermic flora, Ghagotia Union, Gazipur, Bangladesh

\begin{abstract}
Angiospermic flora of Ghagotia Union representing Magnoliopsida (Dicots) and Liliopsida (Monocots) have been inventoried after survey during the years of 2004 and 2005. A total of 187 species have been recorded from the area. These have been assigned to 65 families and 160 genera. Magnoliopsida is represented by 50 families, 113 genera and 133 species, whereas Liliopsida is represented by 15 families, 47 genera and 54 species.
\end{abstract}

\section{Introduction}

Ghagotia Union is located in Kapasia Upazila under Gazipur District, Bangladesh. It lies between $24^{\circ} 07^{\prime}$ and $24^{\circ} 11^{\prime} \mathrm{N}$ latitudes and $90^{\circ} 38^{\prime}$ and $90^{\circ} 42^{\prime} \mathrm{E}$ longitudes. It is about $70 \mathrm{~km}$ north-east from Dhaka. The Union consists of mainly medium high plain land and low plain land. Some small hillocks are also present in the western part of the Union which are covered by natural secondary Sal (Shorea robusta) forest. Other parts are covered by homestead vegetation and cultivated lands.

Ghagotia Union is mainly dominated by the extension of shallow upland soil. Dissected terrace soil is found in some high ridges and alluvial soil is found in the valleys. The western part of the area is composed of some red and brown clay of the mixed variety of deep dissected terrace soil. The eastern part of the area contains some fertile soil with grey and white-brown plain clay soil (Rizvi 1969). The area enjoys a tropical climate characterized by a period of high precipitation from May to October and six months of relatively dry period from November to April. The mean annual rainfall is about $1693 \mathrm{~mm}$. Temperature of the area ranges from $21-34^{\circ} \mathrm{C}$. The maximum temperature was recorded in April and the minimum was recorded in January during the study period.

A number of floristic works have so far been done in greater Dhaka District including Ismail and Mia (1973), Alam (1995), Hossain et al. (1995), Rahman and Hassan (1995) and Rashid et al. (1995). But no floristic studies are found in the Ghagotia Union of Gazipur District. Moreover, the area supports a large number of angiospemic species including herbs, shrubs, trees, climbers, epiphytes, parasites and also plenty of hydrophytes. Like other parts of the country, the floristic elements of this area is in risk

${ }^{1}$ Corresponding author. 
because of various anthropogenic activities including irrigation and modern agriculture, population settlements and firewood collection and also habitat degradation. In order to make a documentation of the angiospermic vegetation of the area, an attempt has been made to prepare a preliminary checklist of the angiospermic plant species occurring in Ghagotia Union of Gazipur District.

\section{Materials and Methods}

The work is based on the fresh materials collected through repeated field trips (eight in total) to the area during the years of 2004 and 2005. Botanical specimens were collected, and field identification of the collected specimens were confirmed comparing with herbarium specimens at Salar Khan Herbarium (University of Dhaka) and Bangladesh National Herbarium. In some cases, standard literature, such as Hooker (1872-1897), Prain (1903), Brandis (1906), Kanjilal et al. (1934, 1938, 1939, 1940), Bor (1960), Khan (1977, 1984, 1985), Deb (1981, 1983), Matthew (1999a, 1999b, 1999c) and Uddin and Hassan (2004) were consulted for identification purposes. The specimens were mounted and deposited in the Salar Khan Herbarium, University of Dhaka for future references.

\section{Results}

In the present survey, a total of 187 angiospermic species under 160 genera and 65 families have been recorded from the Ghagotia Union. Magnoliopsida is represented by 50 families, 113 genera and 133 species, while Liliopsida is represented by 15 families, 47 genera and 54 species. The families have been arranged according to Cronquist (1981). The genera under each family and the species under each genus are arranged in an alphabetical order. For each species, nomenclature has been brought up to date and local name (wherever available) and a short annotation is provided.

\section{MAGNOLIOPSIDA (DICOTS)}

\section{Annonaceae}

Uvaria hamiltonii Hook. f. \& Thomson, Fl. Ind.: 96 (1820). Local name: Bandor kola. A scandent shrub.

\section{Lauraceae}

Litsea glutinosa (Lour.) C.B. Robinson in Philip. J. Sci. Bot. 6: 321 (1911). Sebifera glutinosa Lour., Fl. Cochinch.: 638 (1990). Local name: Chapaitta. A medium-sized, evergreen tree. 


\section{Piperaceae}

Peperomia pellucida (L.) H.B.K., Nov. Gen. et. Sp. 1: 64 (1815). Piper pellucidum L., Sp. Pl. 1: 30 (1753). Local name: Luchipata. A small, annual herb.

\section{Nymphaeaceae}

Nymphaea nouchali Burm. f., Fl. Ind.: 120 (1768). Nymphaea pubescens Willd., Sp. P1. 2: 1154 (1799). Local name: Shapla. A perennial aquatic herb with creeping rhizome.

N. rubra Roxb. ex Salisb., Parad. London 1: sub. t. 14 (1805). Local name: Ogul phul. A perennial aquatic herb with creeping rhizome.

N. stellata Willd., Sp. P1. 2: 1153 (1799). Local name: Shinduk. A perennial aquatic herb with creeping rhizome.

\section{Ulmaceae}

Trema orientalis (L.) B1., Mus. Bot. Lugd.-Bat. 2: 63 (1856). Celtis orientalis L., Sp. P1.: 1044 (1753). Local name: Narsa. An evergreen, small tree.

\section{Moraceae}

Ficus benghalensis L., Sp. P1.: 1059 (1753). Local name: Bot. A large, spreading tree.

F. heterophylla L. f., Suppl.: 442 (1781). Local name: Bhuidumur. A hispid, scandent shrub.

F. rumphii B1., Bijdr.: 437(1825). Local name: Guya assawth. A large tree.

\section{Portulacaceae}

Portulaca oleracea L., Sp. Pl.: 445 (1753). Local name: Bara nunia. A prostrate, annual herb.

\section{Chenopodiaceae}

Chenopodium ambrosioides L., Sp. P1.: 219 (1753). An annual, erect herb.

\section{Amaranthaceae}

Achyranthes aspera L., Sp. Pl. 1: 204 (1753). Local name: Apang. A perennial herb. Alternanthera philoxeroides (Mart.) Griseb., Abh. Ges. Goett. Wiss 24: 36 (1879). Bucholzia phyloxeroides Mart., Beitr. Amar.: 107 (1825). Local name: Helencha. An annual herb.

A. sessilis (L.) R. Br. ex DC., Cat. Hort. Monsp.: 77 (1813). Gomphrena sessilis L., Sp. P1.: 225 (1753). Local name: Kantanotey. An annual, profusely branched herb.

Celosia argentea L., Sp. P1.: 205 (1753). Local name: Thainthainna. An annual, erect herb. 


\section{Polygonaceae}

Persicaria hydropiper (L.) Spach, Hist. Veg. 10: 536 (1841). Polygonum hydropiper L., Sp. Pl.: 361 (1753). Local name: Pakurmul. An annual herb.

P. minor (Huds.) Opiz, Seenam, Rosplin, Kbeteny, Ceske: 72 (1852). Polygonum minus (Huds.), Fl. Angl. 1: 148 (1762). An annual, erect or ascending herb.

Polygonum plebejum R. Br., Prodr. Fl. Nov. Holl.: 420 (1810). A prostrate or diffuse herb.

Rumex maritimus L., Sp. Pl.: 335 (1753). Local name: Ban palang. An annual herb.

\section{Dipterocarpaceae}

Shorea robusta Roxb. ex Gaertn. f., De Fruct. 3: 48, t. 186 (1805). Local names: Gojari, Sal. A tall, deciduous tree.

\section{Tiliaceae}

Microcos paniculata L., Sp. Pl. 1: 514 (1753). Local name: Dattoi. A shrub or small tree.

\section{Malvaceae}

Sida cordata (Burm. f.) Borss. in Blumea 14 (1): 182 (1966). Melochia cordata Burm. f., Fl. Ind.: 143 (1768). Local name: Junka. An annual, prostrate or ascending herb.

Urena lobata L., Sp. P1.: 692 (1753). Local name: Banokra. An undershrub.

\section{Lecythidaceae}

Barringtonia acutangula (L.) Gaertn., Fruct. 2: 97 t. 101 (1791). Eugenia acutangula L., Sp. Pl.: 471 (1753). Local name: Hizol. A small tree.

Careya arborea Roxb., Corom. Pl. 3: 14. t. 218 (1811). Local name: Gadila. A small to medium-sized, deciduous tree.

\section{Flacourtiaceae}

Flacourtia indica (Burm. f.) Merril, Interpr. Rumph. Herb. Amb.: 377 (1917). Gmelina indica Burm. f., Fl. Ind.: 132, t. 39, f. 5 (1768). Local name: Dephoi gota. A much branched, thorny shrub.

F. jangomas (Lour.) Raeusched. Nomencl. Bot. 3: 290 (1797). Stigmarota jangomas Lour., Fl. Cochinch. 2: 634 (1790). Local name: Fela gota. A middle-sized tree.

\section{Cucurbitaceae}

Coccinia grandis (L.) Voit., Hort. Suburb. Calcut.: 59 (1845). Bryonia grandis L., Mant. Pl. 1: 126 (1767). Local names: Kawajhinga,Telakucha. A much branched, climbing or prostrate herb. 


\section{Capparaceae}

Cleome viscosa L., Sp. P1.: 672 (1753). Local name: Hurhuria. An erect, glandularpubescent herb.

\section{Sapotaceae}

Manilkara hexandra (Roxb.) Dub. in Ann. Muss. Col. Marseille, Ser. 3, 3: 9 (1915). Mimusops hexandra Roxb., Pl. Cor. 1: 16, t. 15 (1795). Local name: Khiron gota. A tree with deeply furrowed bark.

\section{Mimosaceae}

Acacia concinna (Willd.) DC., Prodr. 2: 464 (1825). Mimosa concinna Willd., Sp. Pl. 4: 1039 (1805). Local name: Banritha. A prickly shrub.

Albizia chinensis (Osb.) Merr., Amer. J. Bot. 3: 575 (1916). Mimosa chinensis Osb., Degbok Ostind. Resa.: 233 (1757). Local name: Mashkala. A tall, unarmed tree.

A. procera Benth. in Hook., London J. Bot. 3: 89 (1844). Local name: Koroi. A mediumsized tree.

Mimosa pudica L., Sp. Pl.: 518 (1753). Local name: Lojjabati. A prickley, woody herb.

\section{Caesalpiniaceae}

Senna sophera (L.) Roxb., Mem. N.Y. Bot. Gard. 35: 440 (1982). Cassia sophera L., Sp. Pl.: 279 (1753). Local name: Jhingi. A woody herb to undershrub.

\section{Fabaceae (Papilionaceae)}

Crotalaria pallida Aiton, Hort. Kew, 2: 20 (1789). Local name: Bara jhanjhani. An annual herb.

Desmodium heterophyllum (Willd.) DC., Prodr. 2: 334 (1825). Desmodium triflorum Wight. \& Arn., Prodr.: 229 (1834). A procumbent herb.

Erythrina ovalifolia Roxb. [Hort. Beng.: 53 (1814) nom. nud.], Fl. Ind. 3: 251(1832). Local name: Mandar. A deciduous, small tree.

Pueraria phaseoloides (Roxb.) Benth., J. Linn. Soc. Bot. 9: 125 (1867). Dolichos phaseoloides Roxb., Fl. Ind. 3: 316 (1832). A herbaceous, pubescent climber.

\section{Lythraceae}

Lagerstroemia parviflora Roxb., P1. Corm. 1: 47, t. 66 (1795). Local name: Tila jarul. A small, bushy tree.

L. speciosa (L.) Pers., Syn. 2: 72 (1807). Munchausia speciosa L., Mant. Pl. 2: 243 (1771). Local name: Jarul. A large, deciduous tree. 


\section{Myrtaceae}

Syzygium fruticosum (Roxb.) DC., Prodr. 3: 260 (1828). Eugenia fruticosa Roxb., Fl. Ind. 2: 87 (1832). Local name: Titijam. A small tree.

\section{Onagraceae}

Ludwigia adscendens (L.) Hara, J. Jap. Bot. 28: 290 (1953). Jussiaea adscendens L., Mant. 1: 69 (1767). Local name: Keshardam. A creeping or floating herb.

L. hyssopifolia (G. Don.) Exell. Garica de Orta 5: 471 (1957). Jussiaea hyssopifolia G. Don, Gen. Syst. 2: 693 (1832). A branched herb.

\section{Melastomaceae}

Melastoma malabathricum L., Sp. Pl.: 390 (1753). Local name: Datranga. A shrub.

\section{Combretaceae}

Terminalia bellirica (Gaertn.) Roxb., Pl. Corom. 2: 54, t, 198 (1805). Myrobalanus bellirica Gaertn., De. Fruct. Semi. 2: 90, t. 97 (1791). Local name: Bohera. A large tree.

\section{Rhizophoraceae}

Carallia brachiata (Lour.) Merr., Philip. J. Sci. 15: 249 (1919). A small to mediumsized tree with erect trunk.

\section{Alangiaceae}

Alangium salvifolium (L. f.) Wangerin in Pfreich 41: 9 (1910). Grewia salvifolia L. f. Suppl.: 409 (1781). Local name: Gugur. A small tree.

\section{Loranthaceae}

Dendrophthoe falcata (L. f.) Etting. in Denschr. Akad. Wissench. Wien. Mathem Naturawiss. Cl. 32: 52 (1872). Loranthus falcatus L. f., Suppl. Sp. Pl.: 221 (1781). A parasite with terete branchlets.

Macrosolen cochinchinensis (Lour.) van Tiegh., Bull. Soc. B. Fr. 41: 122 (1895). Loranthus cochinchinensis Lour., Fl. Cochin. 1: 195 (1790). Local name: Chhota banda. A stout, parasitic shrub.

\section{Euphorbiaceae}

Antidesma gaesembilla Gaertn., Fruct. 1: 189, t. 39 (1788). Local name: Khudijam. A small tree.

Aporusa dioica (Roxb.) Muell.-Arg. in DC., Prodr. 15(2): 472 (1866). Alnus dioica Roxb., Fl. Ind. 3: 580 (1832). A medium-sized, evergreen tree.

A. wallichii Hook. f., Fl. Brit. Ind. 5: 350 (1885). A medium-sized tree. 
Bridelia retusa (L.) Spreng., Syst. Veg. 3: 48 (1829). Clutia retusa L., Sp. Pl.: 1042 (1753). A medium-sized tree.

Croton bonplandianum Baill., Adansonia 4: 339 (1864). An annual herb.

Gelonium multiflorum Roxb., Fl. Ind. 3: 832 (1832). A medium-sized tree.

Jatropha curcas L., Sp. P1.: 1006 (1753). Local name: Veron. A large, glabrous shrub or rarely small tree.

J. gossypifolia L., Sp. P1.: 1006 (1753). Local name: Lalbherenda. A small shrub.

Macaranga peltata (Roxb.) Muell.-Arg. in DC., Prodr. 15(2): 1010 (1866). Osyris peltata Roxb. (1832). Local name: Pidali. A small tree.

Mallotus philippensis (Lam.) Muell.-Arg. in Linnaea 34: 196 (1865). Croton philippense Lam., Encycl. Meth. B. 2: 209 (1786). A medium-sized tree.

Phyllanthus reticulatus Poir. in Lam., Encycl. Meth. B. 5: 298 (1804). Local name: Sitki. A large, scandent shrub or small tree.

P. urinaria L., Sp. P1.: 982 (1753). An erect, glabrous, annual herb.

Putranjiva roxburghii Wall., Tent. Fl. Nep.: 61 (1826). Local name: Phoolgach. A large tree.

Ricinus communis L., Sp. Pl.: 1007 (1753). Local name: Rerhi. A shrub.

Trewia nudiflora L., Sp. P1. ed. 3: 166 (1753). Local name: Latim. A deciduous tree.

\section{Leeaceae}

Leea aequata L., Syst. Nat. ed. 12, 2: 627 (1767). Leea hirta Roxb., Fl. Ind. 2: 469 (1824). Local name: Pagol gota gach. A shrub.

\section{Sapindaceae}

Allophyllus cobbe B1., Rumph. 3: 131 (1849). Local name: Chitta. A shrub.

Erioglossum rubiginossum (Roxb.) B1., Rumphia 3: 118 (1849). Sapindus rubiginossus Roxb., Fl. Corom. 1: 44. t. 62 (1795). Local name: Hanni gota. A small tree.

\section{Anacardiaceae}

Lannea coromandelica (Houtt.) Merr. J. Arnold. Arbor. 19: 353 (1938). Dialium coromandelicum Houtt., Nat. Hist. 2: 39, t. 5, f. 2 (1774). Local name: Kaphila. A medium-sized, deciduous tree.

\section{Meliaceae}

Aphanamixis polystachya (Wall.) Parker in Ind. For. 57: 486 (1931). Aglaia polystachya Wall. in Roxb., Fl. Ind. 2: 429 (1824). Local name: Roonna. A tree with dense spreading crown. 
Melia azedarach L., Sp. Pl.: 384 (1753). Local name: Gora nim. A medium-sized tree.

\section{Rutaceae}

Clausena suffruticosa Wight \& Arn., Prodr.: 96 (1834). A small shrub.

Glycosmis pentaphylla (Retz.) A. DC., Prodr. 1: 538 (1824). Limonia pentaphylla Retz. Obs. Bot. 5: 24 (1788). Local names: Motkila, Matmoti. A shrub or small tree.

Micromelum minutum (Forst. f.) Wight \& Arn., Prodr. 1: 448 (1834). Limonia minutum Forst. f., Prodr.: 33 (1786). Local name: Thullui. A bushy shrub.

Zanthoxyllum rhetsa (Roxb.) DC. Prodr.: 728 (1824). Fagara rhetsa Roxb. Fl. Ind. 1: 437 (1820). Local name: Bazna. An evergreen, small tree.

\section{Oxalidaceae}

Oxalis corniculata L., Sp. Pl.: 435 (1753). Local name: Amrul. An annual herb.

\section{Apiaceae (Umbelliferae)}

Centella asiatica (L.) Urban in Mart., Fl. Bras. 11. 1: 287 (1879). Hydrocotyle asiatica L., Sp. Pl. : 234 (1753). Local names: Manik pata, Thankuni. A perennial, trailing herb.

\section{Apocynaceae}

Alstonia scholaris (L.) R. Br. in Mem. Wern. Nat. Hist. S. 1: 75 (1811). Echites scholaris L., Mant. Pl. 1: 53 (1767). Local name: Chatim. A medium-sized tree.

Ervatamia coronaria (Jacq.) Stapf, Fl. Trop. Africa 4(1): 127 (1904). Nerium coronarium Jacq., Ic. Pl. Rar. 1: 5, Pl. 52 (1781). Local name: Ban marich. A shrub or small tree.

Holarrhena pubescens (Buch.-Ham.) Wall. ex G. Don, Gen. Syst. 4: 95 (1837). Echites pubescens Buch.-Ham. in Trans. Linn. Soc. 13: 521(1821) Local name: Kudishar. A shrub.

Wrightia arborea (Dennst.) Mabberly in Taxon 26: 533 (1977). Periploca arborea Dennst., Schlüs. H. Malabar.: 13, 23 (1818). Local name: Dhudi. A small, deciduous tree.

\section{Solanaceae}

Nicotiana plumbaginifolia Viv., Elench. Pl. Hort. Dinergo : 26. t. (1802). A slender, erect, annual herb.

Physalis minima L., Sp. Pl.: 183 (1753). An annual, glabrous, herb.

Solanum lasiocarpum Dunal, Hist. Solanum: 222 (1813). Solanum indicum L. Sp. Pl.: 187 (1753). Local name: Titbegun. An under-shrub with prickles.

S. torvum Sw., Nov. Gen. Sp. P1.: 47 (1788). Local name: Bootbegun. A small shrub. 


\section{Convolvulaceae}

Argyreia argentea (Roxb.) Choisy, Mem. Soc. Phys. Geneve. 6: 418 (1833). Lettsomea argentea Roxb., Fl. Ind. ed. Carey \& Wall. 2: 79 (1824). A creeping herb.

Ipomoea aquatica Forsk., Fl. Aeg.-Arab.: 44 (1775). Local name: Kalmilata. A glabrous trailer on water.

I. fistulosa Mart. ex Choisy in DC., Prodr. 9: 349 (1845). Local name: Dholkalmi. A fistular shrub.

Merremia hederacea (Burm. f.) Hallier f., Bot. Jahrb. 18 : 118 (1994). Evolvulus hederaceus Burm. f., Fl. Ind.: 77, t. 30 (1768). Local name: Puhilot. A twinner.

\section{Cuscutaceae}

Cuscuta reflexa Roxb., Pl. Corom. 2: 3, t. 104 (1798). Local name: Swarnalata. A fleshy parasite, forming dense yellow masses on small trees or shrubs.

\section{Boraginaceae}

Heliotropium indicum L., Sp. Pl.: 130 (1753). Local name: Hatisur. An annual, erect herb.

\section{Verbenaceae}

Callicarpa lanceolaria Roxb., Fl. Ind. 1: 395 (1820). A shrub.

Clerodendrum indicum (L.) Kuntze, Rev. Gen. Pl. 2: 506 (1891). Siphonanthus indica L. Sp. Pl. : 109 (1753). A small shrub.

C. viscosum Vent., Gard. Malm. 1: t. 25 (1803). Local name: Vant. A perennial, woody herb to undershrub.

Lippia javanica (Burm. f.) Spreng., Syst. 2: 752 (1825). An undershrub.

Vitex negundo L. Sp. Pl. : 638 (1753). A small tree.

\section{Lamiaceae (Labiatae)}

Anisomeles indica (L.) O. Kuntze, Rev. Gen. : 512 (1891). Nepeta indica L. Sp. Pl. : 571 (1753). Annual or perennial bushy undershrub.

Dysophylla crassicaulis Benth. in Wall., P1. As. Rar. 1.: 30 (1830). An annual herb.

D. verticillata Benth. in Wall., Pl. As. Rar. 1: 30 (1830). An annual herb.

Hyptis suaveolens (L.) Poit., Ann. Mus. Par. 7: 472, t. 29 (1806). Ballota suaveolens L., Syst. Nat. ed. 10: 1100 (1859). Local name: Tokma. An aromatic herb.

Leonurus japonicus Houtt., Nat. Hist. Pl. 9: 366, t. 57 (1778). Leonurus sibiricus Hook. f., Fl. Brit. Ind. 4: 678 (1885). Local name: Roktadron. An erect, stout, leafy herb.

Leucas aspera Spreng., Syst. 2: 743 (1825). Local name: Dorhalash. A herb. 
L. lavandulifolia Sm. in Rees, Cyclop. 20: n. 2 (1819). Local names: Dorhalash, Shetodron. An annual, erect, branched herb.

Ocimum sanctum L., Mart. 1: 85 (1867). Local name: Kalo tulshi. A much branched, soft hairy, perennial herb.

\section{Scrophulariaceae}

Limnophylla heterophylla (Roxb.) Benth., Scroph. Ind.: 25 (1835). Columnea heterophylla Roxb., Fl. Ind. ed. 2, 3: 97 (1832). A glabous, aquatic herb.

Lindernia crustacea (L.) F. Muell., Cens. Austr. P1.: 97 (1882). Capraria crustacea L. Mant. Pl. 1: 87 (1767). A dichotomously branched, prostrate herb.

\section{Bignoniaceae}

Oroxylum indicum (L.) Kurz., For. Fl. Br. Burma 2: 237 (1877). Bignonia indica L., Sp. Pl.: 625 (1753). Local name: Kanaidengi. A medium-sized tree.

\section{Acanthaceae}

Adhatoda zeylanica Medik., Hist. \& Comment. Acad. Elect. Sci. Theod. Palat. 6: 393 (1790). Adhatoda vasica Nees in Wall., Pl. As. Rar. 3: 103 (1832). Local name : Basok. A shrub.

Ecbolium linneanum Kurz. in J. As. Soc. 2: 75 (1871). A shrub.

Justicia gendarusa Burm. f., Fl. Ind.: 10 (1768). Local name: Bishdoloni. An undershrub.

Phlogacanthus curviflorus Nees in DC., Prodr. 11: 320 (1847). A small shrub.

Rungia pectinata (L.) Nees in DC., Prodr. 11: 469 (1847). Justicia pectinata L., Amoen. Acad. 4: 293 (1759). A much branched, prostrate or suberect herb.

Thunbergia grandiflora (Roxb. ex Rottler) Roxb. in Bot. Reg. 6: t. 495 (1820). Flemingia grandiflora Roxb. ex. Rottler, Ges. Naturf. Freund Berlin Neue Schriften 4: 202 (1803). A climber.

\section{Lentibulariaceae}

Utricularia aurea Lour., Fl. Cochinch.1: 26 (1790). Utricularia flexuosa Vahl. Enum. 1: 198 (1804). An aquatic insectivorous herb.

\section{Rubiaceae}

Hymenodictyon excelsum (Roxb.) Wall. in Roxb., Fl. Ind. 2: 149 (1824). Cinchona excelsa Roxb., Fl. Corom. 2: 4. t. 106 (1799). A large tree.

Ixora acuminata Roxb. [Hort. Beng.: 10 (1814) nom nud.], Fl. Ind. 1: 383 (1820). An undershrub. 
I. cuneifolia Roxb., Fl. Ind. 1: 380 (1820). An evergreen shrub.

Morinda angustifolia Roxb. [Hort. Beng. 15 (1814) nom. nud.], Fl. Ind. 2: 201 (1824). An erect shrub.

M. citrifolia L., DC. Prodr. 4: 446 (1830). A glabrous, small tree.

Pavetta tomentosa Roxb. ex Smith in Rees, Cycl. 26: 2 (1819). A shrub.

Xeromphis spinosa (Thunb.) Keay in Bull. Jard. B. Brux. 28: 37 (1958). Gardenia spinosa Thunb., Diss. Gard. n. 7. t. 2 (1780). Locol name: Monkata. A shrub with strong spines.

\section{Asteraceae}

Ageratum conyzoides L., Sp. Pl.: 839 (1753). Local name: Hialmuti. An annual herb. Blumea lacera (Burm. f.) DC. in Wight., Contrib. Bot. Ind.: 14 (1834). Conyza lacera Burm. f., Fl. Ind.: 180, t. 59. (1768). An erect, annual, aromatic herb.

Eclipta prostrata L., Mant. 2: 286 (1771). Local name: Kalokeshoti. An erect, annual herb.

Enhydra fluctuans Lour., Fl. Cochinch.: 511 (1790). Local name: Helencha. A profusely branched, annual herb.

Eupatorium odoratum L., Syst. Nat. ed. 10: 1205 (1759). Local names: Podina, Motmoitta. A shrub.

Gnaphalium luteo-album L., subsp. affine (D. Don) Koster, Blumea 4: 484 (1941). An erect, annual herb.

Grangea maderaspatana (L.) Poir., Enc. Suppl. 2: 825 (1811). Artemisia maderaspatana L., Sp. P1.: 849 (1753). An annual herb.

Mikania cordata (Burm. f.) Robinson in Contrib., Gray Herb. 104: 65 (1934). Eupatorium cordatum Burm. f., Fl. Ind.: 176 (1768). Local name: Assamlata. An annual, twining herb.

Spilanthes calva DC. in Wight, Contrib. Bot. Ind.: 19 (1834). An annual herb.

Synedrella nodiflora (L.) Gaertn., Fruct. 2: 456, t. 171 (1791). Verbesina nodiflora L., Cent. Pl. 1: 28 (1755). A small woody herb.

Xanthium strumarium L., Sp. Pl. : 987 (1753). An erect, annual herb.

\section{LILIOPSIDA (MONOCOTS)}

\section{Hydrocharitaceae}

Blyxa octandra (Roxb.) Planch. ex Thw., Enum. P1. Zeyl.: 332 (1864). Vallisneria octandra Roxb., Pl. Cor. 2 : 34, t. 165 (1802). A stemless, submerged herb. 
Hydrilla verticillata (L. f.) Royle, 111. Bot. Himal. t.: 376. (1839). Serpicula verticillata L. f., Suppl.: 416 (1781). An aquatic herb.

\section{Aponogetonaceae}

Aponogeton natans (L.) Eng. \& Krause in Engl. Pflan. 24: 11 (1906). Saururus natans L., Mant. 2: 227 (1771). An aquatic herb.

\section{Araceae}

Alocasia fornicata (Roxb.) Schott, Oestr. Bot. Wochenbl. 4: 410 (1854). Arum fornicatum Roxb., Fl. Ind. 3: 501 (1832). A tuberous, coarse herb.

Amorphophallus bulbifer (Roxb.) B1., Rumphia 1: 148 (1837). Arum bulbiferum Roxb., Fl. Ind. 3: 510 (1832). Local name: Amla-Bela. A tuberous herb.

Colocasia esculenta (L.) Schott in Schott \& Endl., Melet. Bot.: 18 (1832). Arum esculentum L., Sp. P1.: 965 (1753). Local name: Kachu. A tall coarse herb.

Pistia stratiotes L., Sp. P1.: 963 (1753). Local name: Topapana. A floating, gregarious herb.

Scindapsus officinalis (Roxb.) Schott. in Schott \& Endl. Melet. Bot. 1: 21 (1832). Pothos officinalis Roxb., Fl. Ind. : 431 (1820). A scandent herb.

Typhonium trilobatum (L.) Schott. in Wien., Zeitschr. 3: 72 (1829). Arum trilobatum L., Sp. Pl. : 965 (1753). A tuberous herb.

\section{Commelinaceae}

Commelina benghalensis L., Sp. Pl. : 41 (1753). A slender herb.

Murdania nudiflora (L.) Brenan, Kew Bull. 7: 189 (1952). Commelina nudiflora L., Sp. P1.: 41 (1753). An annual, diffuse herb.

\section{Cyperaceae}

Cyperus difformis L., Cent. Pl. 2: 6 (1756). Local name: Mutha. An annual, tufted herb.

C. diffusus Vahl., Enum. Pl. 2: 321 (1806). A perennial herb.

C. iria L., Sp. Pl. ed. 1. : 45 (1753). An annual or rarely perennial herb.

C. tenuispica Steudl., Syn. Pl. Glumac. 2: 11 (1855). An annual, occasionally short-lived perennial herb.

Eleocharis retroflexa (Poir.) Urban., Symb. Ant. 2: 165 (1900). Scirpus retroflexus Poir (1804). An annual, tufted herb.

Fimbristylis dichotoma (L.) Vahl, Enum. Pl. 2: 287 (1806). Scirpus dichotomus L. Sp. Pl.ed. 1.: 50 (1753). A perennial, rhizomatous herb. 
F. miliacea (L.) Vahl., Enum. Pl. 2: 287 (1806). Scirpus miliaceus L., Syst. Nat. ed. 10: 868 (1759). An annual or occasionally biennial, tufted herb.

F. squarrosa Vahl, Enum. P1. 2: 289 (1806). An annual, tufted herb.

Pycreus pumilus (L.) Nees, Linnaea 9: 283 (1835). Cyperus pumilus L., Cent. Pl. 2: 6 (1756). An annual, densely tufted herb.

Scleria levis Retz., Obs. Bot. 4: 13 (1786). A perennial, rhizomatous herb.

\section{Poaceae (Gramineae)}

Axonopus compressus (Sw.) P. Beauv., Ess. Agrost.: 12(154): 167 (1812). Milium compressum Sw., Prod.: 24 (1788). A tufted, perennial herb.

Bambusa balcooa Roxb., Fl. Ind. 1: 196 (1820). Local name: Barakbans. A tall, stout, densely caespitose bamboo.

Chrysopogon aciculatus (Retz.) Trin., Fund. Agrost. 188 (1820). Andropogon aciculatus Retz. Obs. Bot. 5. 22. (1989). A glabrous herb.

Cynodon dactylon (L.) Pers., Syn. Pl. ed.1.: 85 (1805). Panicum dactylon L., Sp. Pl.: 58 (1753). Local name: Durba. A creeping herb.

Cyrtococcum accrescens (Trin.) Stapf in Hook., Ic. Pl. t.: 3096 (1922). Panicum accrescens Trin., Sp. Gram. Ic. 1, t. 88 (1828). An annual herb.

Echinochloa crusgalli (L.) P. Beauv., Ess. Agrost. 53: 161 (1812). Panicum crusgalli L., Sp. Pl. ed. 1, 1: 56 (1753). Annual or perennial grass.

Eleusine indica (L.) Gaertn., Fruct. 1: 8 (1789). Cynusurus indicus L., Sp. Pl. ed.1.: 72 (1753). A tufted, annual herb.

Eragrostis unioloides (Retz.) Nees ex Steud., Syn. P1. Glum. 1: 264 (1854). Poa unioloides Retz. Obs. Bot. 5: 19 (1789). An annual herb.

Hemarthria protensa Steud., Syn. Pl. Glum. 1: 359 (1854). Local name: Chailla. An erect to decumbent herb.

Hygroryza aristata (Retz.) Nees in Wight \& Arn., Edinb. New Philos. J. 15: 380 (1833) Pharus aristatus Retz., Obs. Bot. 5: 23 (1789). A floating, glabrous herb.

Hymenachne pseudointerrupta C. Mueller, Bot. Zeitung (Berlin). 19: 333 (1861). An annual grass.

Ichananthus vicinus (F. M. Bailey) Merr, Enum. Philipp., Fl. Pl. 1: 70 (1923). Panicum vicinus F.M. Bailey, Syn. Queens., Fl. Suppl. 3: 82 (1890). A perennial herb.

Imperata cylindrica (L.) Reaeschel, Nom. Bot. ed. 3: 10 (1797). Lagurus cylindricus L., Syst. Nat. ed. 10: 878 (1759). Local name: Son. A rhizomatous, perennial grass.

Leptochloa chinensis (L.) Nees, Syll. Pl. Nov. 1: 4 (1824). Poa chinensis L., Sp. Pl. ed. 1: 69 (1753). A tufted grass. 
Melocanna baccifera (Roxb.) Kurz, Prelim. Rep. For. Veg. Pegu. App. B.: 94 (1875). Bambusa baccifera Roxb., Pl. Corm. 3: 38, 243 (1819). Local name: Moli bansh. An evergreen, unarmed bamboo.

Oplismenus compositus (L.) P. Beauv., Ess. Agrost 54:168 (1812). Panicum compositum L., Sp. Pl. ed. 1. : 57 (1753). A perennial grass.

Panicum notatum Retz., Obs. Bot. 4: 18 (1786). A tufted, perennial grass.

P. repens L., Sp. Pl. ed. 2: 87 (1762). A perennial, rhizomatous grass.

Paspalidium flavidum (Retz.) A. Camus in Lecomte, Fl. Gen. Indo-Chine 7: 419 (1922). Panicum flavidum Retz., Obs. Bot. 4: 15 (1786). An annual grass.

Sporobolus diander (Retz.) P. Beauv., Ess. Agrost.: 26: 147 (1812). Agrostis diander Retz., Obs. Bot. 5: 19 (1789). A caespitose, perennial grass.

Vetiveria zizanioides (L.) Nash in Small, Fl. South-East U.S. ed. 1: 67(1903). Phalaris zizanioides L., Mant. 2: 183 (1771). Local name: Binna. A rhizomatous, aromatic, perennial grass.

\section{Zingiberaceae}

Curcuma zedoaria (Christm.) Rosc., Trans. Linn. Soc. London, 8: 354 (1807). Amomum zedoaria Christm. in Christm. \& Panzer, Linn. Pflanesyst. 5: 12 (1779). Local name: Hoiter gach. A stemless herb with pale yellow-white rhizome.

\section{Costaceae}

Costus speciosus (Koenig) Smith in Trans. Linn. Soc. London 1: 249 (1791). Banksea speciosa Koenig in Retz., Obs. Bot. 3: 75 (1783). A rhizomatous herb.

\section{Arecaceae (Palmae)}

Calamus viminalis Willd., Sp. Pl. 2: 203 (1799). A thicket-forming climber.

Caryota urens L., Sp. Pl.: 1189 (1753). A tall, monoecious palm.

\section{Pandanaceae}

Pandanus fascicularis Lam. in Hook, f., Fl. Brit. Ind. 6: 485 (1894). Local name: Keya. A bushy shrub.

\section{Lemnaceae}

Lemna perpusilla Torrey, Fl. New York. 2: 245 (1843). A small, floating aquatic herb.

\section{Pontederiaceae}

Eichhornia crassipes (Mart.) Solms in A. DC., Mon. Phan. 4: 527 (1883). Pontederia crassipes Mart., Nov. Gen. Sp.: 9, t. 4 (1823). Local name: Kachuripana. An aquatic, free-floating herb. 
A PRELIMINARY CHECKLIST OF THE ANGIOSPERMIC FLORA

Monochoria hastata (L.) Solms. in A. DC., Mon. Phan. 4: 523 (1883). Pontederia hastata L., Sp. Pl.: 288 (1753). An aquatic, emergent herb.

\section{Liliaceae}

Curculigo orchioides Gaertn., Fruct. 1: 63, t. 13 (1788). A slender herb with elongated rhizome.

\section{Dioscoreaceae}

Dioscorea bellophylla (Prain) J. O. Voigt ex Haines, Forest Fl. Choto Nagpur.: 530 (1910). Dioscorea nummularia var. bellophylla Prain, Bengal P1. 2: 802 (1903), Ind. Rep. 1963. A perennial climber.

D. pentaphylla L., Sp. Pl.: 1032 (1753). A large, slender twinter.

\section{Orchidaceae}

Vanda tessellata (Roxb.) Hook. ex G. Don in Loud., Hort. Brit.: 372 (1830). Epidendrum tessellatum Roxb., Pl. Corom. 1: 34, t. 42 (1795). An epiphytic herb.

\section{Acknowledgement}

The authors are grateful to Dr. M. Matiur Rahman, Director, Bangladesh National Herbarium for allowing to use herbarium facilities during the study. They also gratefully acknowledge the cooperation rendered by Dr. Mahbuba Khanam, Dr. M. Oliur Rahman and Mr. Sarder Nasir Uddin of Bangladesh National Herbarium particularly for identification of some critical specimens. The authors thank Dr. M. Oliur Rahman also for his comments on an early draft of the manuscript.

\section{References}

Alam, M.K. 1995. Diversity in the woody flora of Sal (Shorea robusta) forest of Bangladesh. Bangladesh J. Forest Sci. 24(1): 41-51.

Brandis, D. 1906. Indian trees (2nd Repr. 1978). Bishen Singh Mahendra Pal Singh, Dehra Dun, 767 pp.

Bor, N.L. 1960. The Grasses of Burma, Ceylon, India and Pakistan (excluding Bambosoideae). Pergamon Press, Oxford.

Cronquist, A. 1981. An integrated system of classification of flowering plants. Columbia University Press, New York, 1262 pp.

Deb, D.B. 1981. The flora of Tripura State 1: 1-50. R. K. Jain, Today \& Tomorrow's Printers and Publishers, New Delhi.

Deb, D.B. 1983. The flora of Tripura State 2: 1-601. R. K. Jain, Today \& Tomorrow's Printers and Publishers, New Delhi.

Hossain, A.B.M.E., Khan, S.A. and Islam, M.A. 1995. An inventory of plant diversity in relation with the ecology and environment of Jahangirnagar University. Vegetational composition and their taxonomic identity. Bangladesh J. Life Sci. 7(1\&2): 95-103. 
Hooker, J.D. 1872-1897. The Flora of British India Vols. 1-7 (Ind. Repr. 1973). Bishen Singh Mahendra Pal Singh, Dehra Dun, India.

Ismail, M. and Mia, M.M.K. 1973. Studies on some deciduous Sal forests of Bangladesh. Ecology of Bangladesh Vegetation 2: 81-103

Kanjilal, U.N., Kanjilal, P.C. and Das, A. 1934. Flora of Assam 1: 1-386 (Ind. Repr. 1982). A von Book Company, Delhi.

Kanjilal, U.N., Kanjilal, P.C. and Das, A. 1938. Flora of Assam 2: 1-409 (Ind. Repr. 1982). A von Book Company, Delhi.

Kanjilal, U.N., Das, A., Kanjilal, P.C. and De, R.N. 1939. Flora of Assam 3: 1-578 (Ind. Repr. 1982). A von Book Company, Delhi.

Kanjilal, U.N., Kanjilal, P.C., De, R.N. and Das, A. 1940. Flora of Assam 4: 1-377 (Ind. Repr. 1982). A Von Book Company, Delhi.

Khan, M.S. 1977. Onagraceae. In: Khan, M.S. (ed.). Flora of Bangladesh. Fasc. 6: 1-10. Bangladesh National Herbarium and Bangladesh Agricultural Research Council, Dhaka.

Khan, M.S. 1984. Dipterocarpaceae. In: Khan, M.S. (ed.). Flora of Bangladesh. Fasc. 25: 1-15. Bangladesh National Herbarium and Bangladesh Agricultural Research Council, Dhaka.

Khan, M.S. 1985. Convolvulaceae. In: Khan, M.S. (ed.). Flora of Bangladesh. Fasc. 30: 1-59. Bangladesh National Herbarium and Bangladesh Agricultural Research Council, Dhaka.

Matthew, K.M. 1999a. The flora of the Palni Hills, South India 1: 1-575. The Rapinat Herbarium, Tiruchirapalli, India.

Matthew, K.M. 1999b. The flora of the Palni Hills, South India 2: 576-1196. The Rapinat Herbarium, Tiruchirapalli, India.

Matthew, K.M. 1999c. The flora of the Palni Hills, South India 3: 1197-1880. The Rapinat Herbarium, Tiruchirapalli, India.

Prain, D. 1903. Bengal Plants. Vols. 1\&2 (Ind. Repr. 1963). Botanical Survey of India, Calcutta.

Rahman, M.O. and Hassan M.A. 1995. Angiospermic flora of Bhawal National Park, Gazipur (Bangladesh). Bangladesh J. Pl. Taxon. 2(1\&2): 47-79.

Rashid, S.H., Rahman, M.M. and Hossain, A.B.M.E. 1995. An inventory of the under growth resources in Chandra Sal forest at Gazipur, Bangladesh. Bangladesh J. Life Sci. 7(1\&2): 111-118.

Rizvi, S.N.H. 1969. Bangladesh District Gazeteers, Dacca. East Pakistan Govt. Press, Dacca.

Uddin, M.Z. and Hassan, M.A. 2004. Flora of Rema-Kalenga Wildlife Sanctuary. IUCN Bangladesh Country Office, Dhaka, Bangladesh, vi+120 pp. 